\title{
VALORIZACIÓN DE RESIDUOS DE FRUTAS PARA COMBUSTIÓN Y PIRÓLISIS
}

\author{
Andrés Felipe Rojas González, Ciliana Flórez Montes
}

${ }^{1}$ Doctor en Ingeniería_Ingeniería Química, Profesor asociado, Grupo de Investigación en Aprovechamiento de Residuos, Departamento de Ingeniería Química, Universidad Nacional de Colombia Sede Manizales, Campus La Nubia, anfrojasgo@unal.edu.co

2Ingeniera Química, Estudiante de Maestría en Ingeniería_Ingeniería Química, Departamento de Ingeniería Química, Universidad Nacional de Colombia Sede Manizales, Campus La Nubia, cflorezm@unal.edu.co

\section{RESUMEN}

El objetivo de este artículo es establecer la valorización de 49 residuos de frutas como posible materia prima en los procesos de combustión y pirólisis. La valorización de los residuos de frutas se plantea a partir del índice de combustibilidad y de las relaciones atómicas $\mathrm{H} / \mathrm{C}$ y O/C (diagrama de Van Krevelen), que se obtienen por análisis próximo, análisis último y poder calorífico. Se encontró que el poder calorífico superior disminuye con el índice de combustibilidad, el cual es mayor en semillas que en cáscaras. También se encontró que a mayor relación atómica $\mathrm{H} / \mathrm{C}$ y O/C, menor es el poder calorífico de los residuos. Se concluyó que los residuos: semillas de naranja, limón y auyama, y cáscaras de chontaduro, que presentan un poder calorífico superior de 15,$86 ; 17,07 ; 17,35$ y $16,58 \mathrm{MJ} / \mathrm{kg}$ y un índice de combustibilidad de 21,$55 ; 14,02$; 11,42 y 17,51, respectivamente. Estos tres residuos se recomiendan como materias primas para una combustión de llama estable, con ignición a baja temperatura y para la obtención de combustibles líquidos y gaseosos por medio de la pirólisis.

Palabras clave: Combustión, diagrama de Van Krevelen, índice de combustibilidad, pirólisis, residuos de frutas

Recibido: 25 de Octubre de 2018. Aceptado: 24 de Abril de 2019

Received: October 25, 2018. Accepted: April 24, 2019

\section{FRUIT WASTE VALORIZATION FOR COMBUSTION AND PYROLYSIS}

\begin{abstract}
The aim of this paper is to establish the valorization of 49 fruit waste as possible raw material in the combustion and pyrolysis process. The valorization of fruit waste is based on the combustibility index and the atomic ratios $\mathrm{H} / \mathrm{C}$ and $\mathrm{O} / \mathrm{C}$ (Van Krevelen diagram), which are obtained by proximate analysis, ultimate analysis and heating value. It was found that the heating value decreases with the combustibility index, which is higher in seeds than in peels. Also, it was found that for a higher atomic ratio $\mathrm{H} / \mathrm{C}$ and O/C, the heating value of waste is lower. It was concluded that the waste: orange, lemon and auyama seeds, and chontaduro peels, which have a higher calorific value of 15,86; 17,07; 17,35 and 16,58, and a combustibility index of 21.55; 14,02; 17.51 and 11,42, respectively. These three wastes are recommended as raw materials for a stable flame combustion, with low ignition temperature and for the obtaining of liquid and gaseous fuels through the pyrolysis.
\end{abstract}

Keywords: Combustion, combustibility index, fruits waste, pyrolysis, Van Krevelen diagram

Cómo citar este artículo: R. Rojas, C. FLorez. "Valorización de residuos de frutas para combustión y pirólisis", Revista Politécnica, vol. 15, no.28 pp.42-53, 2019. DOI: https://doi.org/10.33571/rpolitec.v15n28a4 


\section{INTRODUCCIÓN}

En Colombia, las industrias del procesamiento de frutas generan aproximadamente 71.943.813 toneladas de residuos sólidos vegetales [1] que tienen un fuerte impacto sobre el medio ambiente, ya que presentan altos contenidos de materia orgánica, elementos minerales y presencia de patógenos vegetales que contaminan el aire, suelo y agua [2]. En el procesamiento de frutas, usualmente se obtienen residuos como cáscaras, semillas, hojas, vástagos y capachos o estopa, los cuales se han convertido en un problema no solo ambiental sino económico relacionado con los costos de disposición (embalaje, almacenamiento, transporte, neutralización, recepción en rellenos sanitarios, y/o incineración, entre otros) [3]. El costo por tonelada de residuos sólidos, en algunos países latinoamericanos, se encuentra entre 30 y 80 US $\$$ en recolección, de 10 a 20 US $\$$ en transporte y 10 a 20 US $\$$ en disposición final en rellenos [4].

En este estudio se hace referencia exclusivamente a residuos de frutas, que corresponden a un tipo de biomasa generado en las labores de campo de cultivos durante la cosecha y clasificación, en la etapa de almacenamiento y transporte o en los procesos agroindustriales. El aprovechamiento de esta biomasa favorece de forma significativa la conservación del medio ambiente, ya que su contenido energético procede de la energía solar fijada en el proceso fotosintético [5]. Generalmente, este tipo de biomasa es usada como alimentación animal, abono y biofertilizantes [6]. Sin embargo, se han desarrollado otros procesos como la conversión bioquímica y termoquímica, y la extracción física, que buscan incrementar el valor de la biomasa produciendo compuestos químicos, energía, biocombustibles, carbón activado, entre otros productos [7].

La obtención de energía mediante combustión directa y la producción de combustibles sólidos, líquidos y gaseosos por medio del proceso de pirólisis, son algunas alternativas de aprovechamiento energético de la biomasa [8]. El índice de combustibilidad (IC) representa un parámetro muy importante al momento de evaluar el potencial aprovechamiento de un residuo como combustible. Este índice se determina por el cociente entre el porcentaje en masa de materia volátil (MV) y el porcentaje en masa de carbono fijo (CF).

$$
I C=\frac{M V}{C F}
$$

Este índice, el cual es adimensional, se emplea para determinar la capacidad que tiene un material combustible para mantener 0 no un proceso de combustión en determinadas condiciones. A través de este parámetro es posible comparar el potencial energético de diferentes materiales, de tal manera que a mayor sea el IC, mejor será la combustión [9, 10]. Esto se traduce en una mayor estabilidad de la llama y temperaturas bajas de ignición del material que se está quemando. Prieto y Chaves [9] demostraron la existencia de una relación entre eventos térmicos y parámetros cinéticos, encontrando una relación inversamente proporcional entre la energía de activación media ponderada aparente (Em) y el IC. Es decir, entre mayor sea este índice menor será la energía de activación media ponderada aparente $y$, por tanto, la muestra analizada será más reactiva en procesos de combustión.

Además, el diagrama de Van Krevelen, que muestra la relación atómica de Hidrógeno/Carbono frente a la relación atómica de Oxígeno/Carbono (H/C vs. O/C), hace posible predecir y comparar el poder calorífico de diferentes materiales. Este gráfico permite establecer que el poder calorífico aumenta conforme disminuye la relación atómica O/C y aumenta la relación atómica $\mathrm{H} / \mathrm{C}$ [11-13]. Este diagrama también es utilizado para conocer que material se puede utilizar para el proceso de pirólisis con el fin de obtener en mayor medida un combustible sólido (biosólido o biochar), líquido (bioaceite) o gaseoso [8].

El objetivo de este trabajo es establecer la valorización de residuos de frutas (cáscaras, semillas, vástagos y capacho o estopa de coco) como materia prima en los procesos de combustión y pirólisis, a través de la caracterización por análisis próximo, último y de poder calorífico. Este potencial de aprovechamiento se plantea a partir del índice de combustibilidad y de las relaciones atómicas $\mathrm{H} / \mathrm{C}$ y O/C según el diagrama de Van Krevelen. 


\section{MATERIALES Y MÉTODOS}

\section{Obtención y pretratamiento de los residuos.}

Los residuos de frutas fueron obtenidos de supermercados, tiendas y empresas agroindustriales en el departamento de Caldas Colombia - Sur América. Se seleccionaron 49 residuos de 25 frutas entre cáscaras (24), semillas (22), vástagos (2) y capacho o estopa (1). Esta distribución se llevó a cabo debido a que se tiene mayos disponibilidad de estos residuos. Las frutas seleccionadas fueron: guanábana, mango, naranja, maracuyá, tomate de árbol, mandarina, coco, piña, lulo, limón, zapote, chontaduro, mamoncillo, sandía, papaya, durazno, arazá, curuba, mangostino, guayaba, granadilla, banano, madroño, melón y auyama. Una vez adquiridos y separados los residuos se redujeron de tamaño a través de un proceso manual de corte y se sometieron a secado a $45^{\circ} \mathrm{C}$ por $60 \mathrm{~h}$ o hasta alcanzar peso constante, según la norma NREL/TP-540-42620. Posterior al secado, las muestras se molieron en un molino de discos hasta alcanzar un tamaño de partícula pasante malla 60 de la serie Tyler (tamaño menor a $0,246 \mathrm{~mm}$ ).

\section{Caracterización de los residuos.}

Los residuos de frutas se caracterizaron por medio del análisis próximo, análisis último y poder calorífico. Mediante el análisis próximo se determina el contenido porcentual de humedad total $(\mathrm{Ht})$, el cual se determina considerando la humedad a $45^{\circ} \mathrm{C}$ y a $105^{\circ} \mathrm{C}$, como lo establece la norma ASTM E871, materia volátil (MV) por medio de la norma ASTM E872, cenizas (Cz) a través de la ASTM NREL/TP-510-42622 y carbono fijo (CF) de los residuos, el cual se calcula por diferencia. Los análisis se realizan por duplicado utilizando aproximadamente $1 \mathrm{~g}$ de muestra.

El análisis último o elemental se lleva a cabo en un analizador Leco $\mathrm{CHN}-628$ para determinar el contenido de carbono $(\mathrm{C})$, hidrógeno $(\mathrm{H})$ y nitrógeno (N) según la norma ASTM D5373-08, y en un Leco S-632 para cuantificar azufre (S) de acuerdo con la norma ASTM D4239. En los dos equipos se emplea una cantidad de muestra de $0,1 \mathrm{~g}$. Este análisis se realiza por duplicado. El oxígeno $(\mathrm{O})$ se determina por diferencia. Se agruparon los valores de $\mathrm{N}$ y $\mathrm{S}(\mathrm{N}+\mathrm{S})$ para analizar el potencial contaminante de los residuos respecto a las posibles emisiones generadas (NOx y SOx) por estos elementos al ser quedos. Con el análisis elemental se determinó la formula empírica de cada uno de los residuos.

La determinación del poder calorífico superior (PCS) se realiza utilizando $0,1 \mathrm{~g}$ de muestra en una bomba calorimétrica marca Leco AC-350, siguiendo la norma ASTM 5865. En este análisis la muestra se somete a combustión completa en un ambiente adiabático, se registra el cambio de temperatura que se genera. El PCS se obtiene de esta información, considerando que se corrige por contenido de humedad y por contenido azufre [14].

\section{Valorización de los residuos de frutas en combustión y pirólisis}

Con el fin de establecer la valorización de los residuos de frutas en los procesos de combustión y pirólisis, se plantean como parámetros a evaluar el índice de combustibilidad y las relaciones atómicas $\mathrm{H} / \mathrm{C}$ y $\mathrm{O} / \mathrm{C}$ a través del diagrama de Van Krevelen. El índice de combustibilidad toma los resultados del análisis próximo y calcula la relación materia volátil (MV) / carbono fijo (CF), (MV/CF).

El diagrama de Van Krevelen se emplea como base para establecer el potencial aprovechamiento de los residuos de fruta como materia prima en los procesos de combustión y pirólisis [8]. Este diagrama se construye con las relaciones atómicas $\mathrm{H} / \mathrm{C}$ y $\mathrm{O} / \mathrm{C}$, las cuales están dadas por medio de las siguientes ecuaciones (2)-(3) [8]:

$\frac{H}{C}=\frac{\left(\frac{\% H}{M_{H}}\right)}{\left(\frac{\% C}{M_{C}}\right)}$

$\frac{o}{C}=\frac{\left(\frac{\% O}{M_{O}}\right)}{\left(\frac{\% C}{M_{C}}\right)}$

Donde $\% \mathrm{H}$ es el porcentaje en masa de hidrógeno, $\% \mathrm{O}$ es el porcentaje en masa de oxígeno, \%C es el porcentaje en masa de carbono, todos en base seca libre de ceniza. $\mathrm{MH}$, $\mathrm{MO}$ y $\mathrm{MC}$ es el peso atómico del hidrógeno, oxígeno y carbono, respectivamente. Según este diagrama, valores altos de las relaciones $\mathrm{H} / \mathrm{C}$ y O/C favorecen la producción de volátiles y 
líquidos durante el proceso de pirólisis, mientras que con bajos valores de estas relaciones se favorece la producción de sólidos durante la pirólisis. En combustión se busca que la relación de O/C sea baja y el valor de $\mathrm{H} / \mathrm{C}$ sea alto, lo cual garantiza que el residuo tiene un alto poder calorífico que beneficia la obtención de energía [8].

\section{RESULTADOS Y DISCUSIÓN}

\section{Caracterización de los residuos}

Las Tablas 1 y 2 muestras los resultados del análisis próximo completo y del análisis elemental, respectivamente, obtenidos experimentalmente en este trabajo. La Tabla 1 presenta los resultados del análisis próximo en base seca, índice de combustibilidad (MV/CF) y poder calorífico superior (PCS) de los 49 residuos de frutas (entre cáscaras, semillas, vástagos y estopa). Se observa que el contenido de cenizas $(\mathrm{Cz})$ de estos residuos esta entre $0,65 \%$ (cáscara de coco) y $17,88 \%$ (cáscara de melón). De igual forma se aprecia que las semillas presentan un menor contenido de $\mathrm{Cz}$ (por debajo del 5\%), comparado con las cáscaras. El contenido de MV varía entre $71,08 \%$ (cáscara de naranja) y 93,14\% (cáscara de chontaduro). En general, se observa que las cáscaras reportan menor contenido de MV comparado con las semillas. Esto posiblemente se debe a que parte de la MV presente en la mayoría de las cáscaras es de bajo peso molecular (con baja temperatura de evaporación), mientras que en las semillas se presenta MV de alto peso molecular (con altas temperatura de evaporación) [15].

Respecto al carbono fijo, en la Tabla 1 se muestra que varía entre 4,29\% (semilla de naranja) y $27.01 \%$ (cáscara de mangostino). En este parámetro no se aprecia diferencia entre las semillas y las cáscaras. En cuanto al IC, este se encuentra entre $2,64 \%$ (cáscara de mangostino) y $21,55 \%$ (semilla de naranja). El PCS varía entre $15,15 \mathrm{MJ} / \mathrm{kg}$ (cáscara de melón) y 19,89MJ/kg (cáscara de coco). Este resultado confirma la premisa que a mayor contenido de cenizas menor es el poder calorífico de un material [9]. De los residuos analizados, los resultados del análisis próximo para la estopa de coco, el vástago de piña y el vástago de tomate de árbol, se encuentran entre los valores de los análisis próximos obtenidos para las cáscaras y las semillas estudiadas. Al comparar el análisis próximo con datos de trabajos anteriores, se observa que los resultados del presente estudio se encuentran dentro de los rangos reportados en la literatura. Sin embargo, en el caso de la ceniza, se han reportado valores superiores a los encontrados para los residuos analizados [16, 17]. De igual forma, los valores del poder calorífico de los residuos de fruta analizados son congruentes con otros datos presentados en la literatura $[8,16,17]$.

En la Tabla 2 se muestran los resultados del análisis último en base seca libre de cenizas, las relaciones atómicas $\mathrm{H} / \mathrm{C}$ y $\mathrm{O} / \mathrm{C}$, y las fórmulas empíricas de 49, residuos de frutas (entre cáscaras, semillas, vástagos y estopa). Del análisis último se encuentra que el contenido de carbono varía entre $45,21 \%$ (cáscara de melón) y $50,65 \%$ (cáscara de mangostino). En términos generales, se encontró que las cáscaras tienen un mayor contenido de carbono que las semillas. El contenido de hidrógeno no presenta grandes diferencias entre cada uno de los residuos, este varía entre 5,98\% (estopa y cáscara de coco) y $6,21 \%$ (cáscara de melón). Para el contenido de oxígeno en los residuos de frutas, se observa que está entre 43,32 (cáscara de mangostino) y 46,25 (semilla de naranja).

El contenido de nitrógeno y azufre $(\mathrm{N}+\mathrm{S})$ se encuentra entre 0,04 (cáscara de mangostino) y $3,13 \%$ (cáscara de melón). Este parámetro es de gran importancia en combustión, dado que la producción de emisiones tipo NOx y SOx, que causan problemas ambientales, depende del contenido de $\mathrm{N}+\mathrm{S}$ presente en el material empleado como combustible. Por lo tanto, partiendo del contenido de $\mathrm{N}+\mathrm{S}$, se espera que al someter a combustión residuos como el vástago de tomate de árbol $(2,18 \%)$, cáscara de sandía $(2,42 \%)$ y cáscara de melón $(3,13 \%)$, se presenten emisiones considerables de NOx y SOx; mientras que al quemar residuos como cáscara de mangostino $(0,04 \%)$, semilla de curuba $(0,17 \%)$, cáscara $(0,17 \%)$ y estopa de coco $(0,18 \%)$, y cáscara de naranja $(0,41 \%)$, no se esperan emisiones considerables de dichos gases contaminante [3].

Los porcentajes de carbono, hidrógeno, nitrógeno y azufre obtenidos en este estudio para cada uno de los residuos son similares a los datos reportados en la literatura para un conjunto de residuos de fruta, mientras que el porcentaje 
de oxígeno es más alto para los residuos analizados.

Tabla 1. Análisis próximo en base seca, índice de combustibilidad y PCS de residuos de frutas.

\begin{tabular}{|c|c|c|c|c|c|c|}
\hline Residuo de Fruta & $\mathrm{Ht}(\%)$ & $\begin{array}{l}\mathrm{Cz} \\
(\%)\end{array}$ & MV (\%) & $\begin{array}{l}\text { CF } \\
(\%)\end{array}$ & IC & PCS (MJ/kg) \\
\hline $\begin{array}{ll}\text { Cáscara } & \text { de } \\
\text { guanábana } & \end{array}$ & 78,12 & 6,22 & 73,07 & 20,71 & 3,53 & 18,83 \\
\hline Semilla & & & & & & \\
\hline $\begin{array}{l}\text { guanábana } \\
\text { Cáscara }\end{array}$ & 62,48 & 1,58 & 88,28 & 10,14 & 8,71 & 18,24 \\
\hline mango & 73,85 & 3,29 & 82,50 & 14,22 & 5,80 & 18,70 \\
\hline Semilla de mango & 54,36 & 1,36 & 83,77 & 14,87 & 5,63 & 19,05 \\
\hline $\begin{array}{l}\text { Cáscara de } \\
\text { narania }\end{array}$ & 7127 & 406 & 7108 & 2486 & & \\
\hline Semilla de naranja & 63,70 & $\begin{array}{l}\begin{array}{l}4,00 \\
3,26\end{array} \\
\end{array}$ & 92,45 & $\begin{array}{c}24,86 \\
4,29\end{array}$ & $\begin{array}{l}2,86 \\
21,55\end{array}$ & $\begin{array}{l}19,34 \\
15,86\end{array}$ \\
\hline $\begin{array}{ll}\text { Cáscara de } \\
\text { maracuyá }\end{array}$ & 88,99 & 8,03 & 78,25 & 13,72 & 5,70 & 18,04 \\
\hline $\begin{array}{l}\text { Semilla } \\
\text { maracuyá }\end{array}$ & 80,46 & 1,36 & 80,98 & 17,66 & 4,59 & 19,35 \\
\hline $\begin{array}{l}\text { Cáscara de } \\
\text { tomate de árbol }\end{array}$ & 80,41 & 8,67 & 78,82 & 12,51 & 6,30 & 17,81 \\
\hline $\begin{array}{l}\text { Semilla de tomate } \\
\text { de árbol }\end{array}$ & 78.50 & 4.03 & 81,60 & 14.37 & 5,68 & 18,62 \\
\hline Vástago de tomate & 7487 & 128 & 7748 & 972 & 797 & 1683 \\
\hline $\begin{array}{l}\text { Cáscara de } \\
\text { mandarina }\end{array}$ & 74.24 & 3.62 & 77.10 & 19.28 & 4.00 & 1912 \\
\hline $\begin{array}{l}\text { Semilla de } \\
\text { mandarina }\end{array}$ & 67,01 & 1,92 & 86,45 & 11,63 & 7,43 & 18,49 \\
\hline de coco & 74,94 & 3,46 & 73,51 & 23,03 & 3,19 & 19,36 \\
\hline Cáscara de coco & 54,25 & 0,65 & 77,44 & 21,91 & 3,53 & 19,89 \\
\hline Cáscara de piña & 88,57 & 4,81 & 81,16 & 14,03 & 5,78 & 18,48 \\
\hline Vástago de piña & 81,03 & 4,57 & 82,74 & 12,69 & 6,52 & 18,33 \\
\hline Cáscara de lulo & 84,66 & 6,06 & 85,63 & 8,31 & 10,30 & 17,27 \\
\hline Semilla de lulo & 71,28 & 2,30 & 89,28 & 8,42 & 10,60 & 17,71 \\
\hline Cáscara de limón & 79,34 & 3,14 & 83,02 & 13,84 & 6,00 & 18,67 \\
\hline Semilla de limón & 64,50 & 2,07 & 91,41 & 6,52 & 14,02 & 17,07 \\
\hline Cáscara de zapote & 87,15 & 9,16 & 76,48 & 14,36 & 5,33 & 17,96 \\
\hline Semilla de zapote & 58,53 & 2,48 & 86,78 & 10,74 & 8,08 & 18,25 \\
\hline $\begin{array}{l}\text { Cáscara de } \\
\text { chontaduro }\end{array}$ & 63,24 & 1,54 & 93,14 & 5,32 & 17,51 & 16,58 \\
\hline $\begin{array}{l}\text { Semilla } \\
\text { chontaduro }\end{array}$ & 40,85 & 1,37 & 84,95 & 13,68 & 6,21 & 18,90 \\
\hline $\begin{array}{l}\text { Cáscara } \\
\text { mamoncillo }\end{array}$ & 66,99 & 5,23 & 77,12 & 17,65 & 4,37 & 18,78 \\
\hline $\begin{array}{l}\text { Semilla } \\
\text { mamoncillo }\end{array}$ & 59,54 & $\begin{array}{l}1,33 \\
15,1\end{array}$ & 83,54 & 15,13 & 5,52 & 19,09 \\
\hline $\begin{array}{l}\text { Cáscara de sandía } \\
\text { Semilla de sandía }\end{array}$ & 92,45 & 3 & 73,28 & $\begin{array}{l}11,59 \\
8,80\end{array}$ & 6,32 & $\begin{array}{l}16,84 \\
1770\end{array}$ \\
\hline $\begin{array}{l}\text { Semilla de sandía } \\
\text { Cáscara de }\end{array}$ & 36,46 & 2,59 & 88,61 & 8,80 & 10,07 & 17,78 \\
\hline $\begin{array}{l}\text { papaya } \\
\text { Semillas }\end{array}$ & 91,34 & 7,44 & 80,59 & 11,97 & 6,73 & 17,88 \\
\hline papaya & 88,07 & 7,48 & 84,07 & 8,45 & 9,95 & 17,15 \\
\hline durazno & 87,64 & 4,23 & 79,00 & 16,77 & 4,71 & 18,84 \\
\hline $\begin{array}{l}\text { Semilla } \\
\text { durazno }\end{array}$ & 57,59 & 1,27 & 83,03 & 15,70 & 5,29 & 19,17 \\
\hline
\end{tabular}




\begin{tabular}{|c|c|c|c|c|c|c|}
\hline & & \multirow{2}{*}{$\begin{array}{l}2,23 \\
1,74\end{array}$} & \multirow{2}{*}{$\begin{array}{l}77,35 \\
81,64\end{array}$} & \multirow{2}{*}{$\begin{array}{l}20,42 \\
16,62\end{array}$} & \multirow{2}{*}{$\begin{array}{l}3,79 \\
4,91\end{array}$} & \multirow{2}{*}{$\begin{array}{l}19,41 \\
19,18\end{array}$} \\
\hline & & & & & & \\
\hline $\begin{array}{l}\text { Semilla de arazá } \\
\text { Cáscara de } \\
\text { curuba }\end{array}$ & $\begin{array}{l}92,76 \\
55,22\end{array}$ & 4,00 & 73,85 & 22,15 & 3,33 & 19,23 \\
\hline Semilla de curuba & 62,94 & 1,02 & 76,13 & 22,85 & 3,33 & 19,81 \\
\hline \\
\hline $\begin{array}{ll}\text { Cáscara } & \text { de } \\
\text { mangostino } & \end{array}$ & 63,80 & 1,60 & 71,39 & 27,01 & 2,64 & 19,86 \\
\hline \multirow{3}{*}{$\begin{array}{l}\text { Semilla } \\
\text { mangostino } \\
\text { Semilla } \\
\text { guayaba }\end{array}$} & 68,50 & 4,01 & 84,77 & 11,22 & 7,56 & 18,16 \\
\hline & & & & & & \\
\hline & 69,72 & 1,38 & 84,38 & 14,24 & 5,93 & 18,97 \\
\hline $\begin{array}{l}\text { Cáscara } \\
\text { granadilla }\end{array}$ & 76,02 & 4,83 & 85,20 & 9,97 & 8,55 & 17,82 \\
\hline \multirow{4}{*}{$\begin{array}{l}\text { Semilla } \\
\text { granadilla } \\
\text { Cáscara } \\
\text { banano } \\
\text { Cáscara } \\
\text { madroño } \\
\text { Semilla } \\
\text { madroño }\end{array}$} & 38,23 & $\begin{array}{l}2,78 \\
10,6\end{array}$ & 80,22 & 17.00 & 4,72 & 19,06 \\
\hline & 88,87 & 8 & 75,77 & 13,55 & 5,59 & 17,68 \\
\hline & 83,32 & 4,75 & 79,91 & 15,34 & 5,21 & 18,64 \\
\hline & 60,19 & $\begin{array}{l}1,36 \\
17,8\end{array}$ & 85,33 & 13,31 & 6,41 & 18,29 \\
\hline \multirow{2}{*}{$\begin{array}{l}\text { Cáscara de melón } \\
\text { Semilla de melón }\end{array}$} & 95,57 & 8 & 76,11 & 6,01 & 12,66 & 15,15 \\
\hline & 55,88 & 3,47 & 86,50 & 10,03 & 8,62 & 17,99 \\
\hline \multirow{2}{*}{$\begin{array}{l}\text { Cáscara } \\
\text { auyama } \\
\text { Semilla } \\
\text { auyama }\end{array}$} & 88,05 & 6,22 & 84,65 & 9,13 & 9,27 & 17,47 \\
\hline & 45,08 & 3,73 & 88,52 & 7,75 & 11,42 & 17,35 \\
\hline
\end{tabular}

Ht: humedad total, Cz: ceniza, MV: materia volátil, CF: carbono fijo, MV/CF: índice de combustibilidad, PCS: poder calorífico superior. Fuente: Propia de los autores.

Tabla 2. Análisis último en base seca libre de cenizas y relaciones atómicas de residuos de frutas

\begin{tabular}{|c|c|c|c|c|c|c|c|}
\hline Residuo de Fruta & C (\%) & $\begin{array}{l}\mathrm{H} \\
(\%)\end{array}$ & $\mathrm{O}(\%)$ & $\begin{array}{l}\mathrm{N}+\mathrm{S} \\
(\%)\end{array}$ & $\mathrm{H} / \mathrm{C}$ & $\mathrm{O} / \mathrm{C}$ & Fórmula empírica \\
\hline Cáscara & & & & & & & $\mathrm{C}_{20} \mathrm{H}_{29,2} \mathrm{O}_{13,4} \mathrm{~N}_{0,17} \mathrm{~S}_{0,05}$ \\
\hline & 49,23 & 6,04 & 43,90 & 0,83 & 1,47 & 0,67 & \\
\hline $\begin{array}{l}\text { Semilla } \\
\text { guanábana }\end{array}$ & & 6,01 & & 0,81 & & & $\mathrm{C}_{20} \mathrm{H}_{30,0} \mathrm{O}_{14,3} \mathrm{~N}_{0,16} \mathrm{~S}_{0,06}$ \\
\hline Cáscara de mango & & 6,02 & & 0,80 & & & $\mathrm{C}_{20} \mathrm{H}_{29,7} \mathrm{O}_{14,0} \mathrm{~N}_{0,13} \mathrm{~S}_{0,07}$ \\
\hline Semilla de mango & 48,5 & 6,00 & 44,93 & 0,5 & 1,48 & 0,69 & $\mathrm{C}_{20} \mathrm{H}_{29,4}$ \\
\hline de naranja & 50,13 & 6,01 & & 0,41 & 1,44 & 0,65 & $\mathrm{C}_{20} \mathrm{H}_{28,5} \mathrm{O}_{13,0} \mathrm{~N}_{0,09} \mathrm{~S}_{0,02}$ \\
\hline Semilla de naranja & & 6,03 & & 1,27 & 1,56 & 0,75 & $\mathrm{C}_{20} \mathrm{H}_{30,9} \mathrm{O}_{14,9} \mathrm{~N}_{0,33} \mathrm{~S}_{0,06}$ \\
\hline $\begin{array}{l}\text { Cáscara } \\
\text { maracuyá }\end{array}$ & & 6.07 & & 1.38 & 1.52 & 0.70 & $\mathrm{C}_{20} \mathrm{H}_{30,2} \mathrm{O}_{14,1} \mathrm{~N}_{0,33} \mathrm{~S}_{0,07}$ \\
\hline Semi & & & & & & & $\mathrm{C}_{20} \mathrm{H}_{29,1} \mathrm{O}_{13,7} \mathrm{~N}_{0,09} \mathrm{~S}_{0,03}$ \\
\hline $\begin{array}{l}\text { maracuyá } \\
\text { Cáscara de tomate }\end{array}$ & 49,00 & 6,00 & 44,56 & 0,44 & 1,47 & 0,68 & $\mathrm{C}_{20} \mathrm{H}_{30,4} \mathrm{O}_{14,2} \mathrm{~N}_{0,44} \mathrm{~S}_{0,04}$ \\
\hline de árbol & 47,50 & 6,08 & 44,91 & 1,51 & 1,54 & 0,71 & \\
\hline $\begin{array}{l}\text { Semilla de tomate } \\
\text { de árbol }\end{array}$ & 48,22 & 6,03 & 44,87 & 0,88 & 1,50 & 0,70 & $\mathrm{C}_{20} \mathrm{H}_{29,7} \mathrm{O}_{14,0} \mathrm{~N}_{0,20} \mathrm{~S}_{0,05}$ \\
\hline $\begin{array}{l}\text { Vástago de tomate } \\
\text { de árbol }\end{array}$ & 46,56 & 6,14 & 45,12 & 2,18 & 1,58 & 0,73 & $\mathrm{C}_{20} \mathrm{H}_{31,4} \mathrm{O}_{14,5} \mathrm{~N}_{0,70} \mathrm{~S}_{0,05}$ \\
\hline Cáscara & & & & & & & $\mathrm{C}_{20} \mathrm{H}_{29,1} \mathrm{O}_{13,5} \mathrm{~N}_{0,13} \mathrm{~S}_{0,04}$ \\
\hline $\begin{array}{l}\text { mandarina } \\
\text { Semilla }\end{array}$ & 49,14 & 6,02 & 44,23 & 0,61 & 1,47 & 0,68 & $\mathrm{C}_{20} \mathrm{H}_{29,9} \mathrm{O}_{14,2} \mathrm{~N}_{0,18} \mathrm{~S}_{0.05}$ \\
\hline mandarina & 47,88 & 6,01 & 45,32 & 0,80 & 1,51 & 0,71 & \\
\hline
\end{tabular}




\begin{tabular}{|c|c|c|c|c|c|c|c|}
\hline \multirow{2}{*}{$\begin{array}{l}\text { Estopa o capacho } \\
\text { de coco }\end{array}$} & & & & & & & $\mathrm{C}_{20} \mathrm{H}_{28,6} \mathrm{O}_{13,3} \mathrm{~N}_{0,04} \mathrm{~S}_{0,01}$ \\
\hline & 49,80 & 5,98 & 44,04 & 0,18 & 1,44 & 0,66 & \\
\hline \multirow{2}{*}{$\begin{array}{l}\text { Cáscara de coco } \\
\text { Cáscara de piña }\end{array}$} & 49,80 & 5,98 & 44,05 & 0,17 & 1,44 & 0,66 & $\mathrm{C}_{20} \mathrm{H}_{28,6} \mathrm{O}_{13,5} \mathrm{~N}_{0,03} \mathrm{~S}_{0,02}$ \\
\hline & 48,10 & 6,04 & 44,88 & 0,98 & 1,51 & 0,70 & $\mathrm{C}_{20} \mathrm{H}_{29,9} \mathrm{O}_{14,0} \mathrm{~N}_{0,23} \mathrm{~S}_{0,05}$ \\
\hline Vástago de piña & 47,87 & 6,04 & 45,08 & 1,01 & 1,51 & 0,71 & $\mathrm{C}_{20} \mathrm{H}_{30,0} \mathrm{O}_{14}$ \\
\hline Cáscara de lulo & 46,93 & 6,06 & 45,61 & 1,40 & 1,55 & 0,73 & $\mathrm{C}_{20} \mathrm{H}_{30,7} \mathrm{O}_{14,6} \mathrm{~N}_{0,33} \mathrm{~S}_{0,08}$ \\
\hline Semilla de lulo & 47,28 & 6,01 & 45,73 & 0,98 & 1,53 & 0,73 & $\mathrm{C}_{20} \mathrm{H}_{30,2} \mathrm{O}_{14,5} \mathrm{~N}_{0}$, \\
\hline Cáscara de limón & 48,20 & 6,02 & 44,98 & 0,79 & 1,50 & 0,70 & $\mathrm{C}_{20} \mathrm{H}_{29,7} \mathrm{O}_{14,0} \mathrm{~N}_{0,21}$ \\
\hline Semilla de limón & 46,96 & 6,01 & 45,99 & 1,03 & 1,54 & 0,73 & $\mathrm{C}_{20} \mathrm{H}$ \\
\hline Cáscara de zapote & 47,80 & 6,09 & 44,63 & 1,49 & 1,53 & 0,70 & $\mathrm{C}_{20} \mathrm{H}_{3}$ \\
\hline \multirow{2}{*}{$\begin{array}{l}\text { Semilla de zapote } \\
\text { Cáscara de }\end{array}$} & 47,68 & 6,02 & 45,42 & 0,88 & 1,51 & 0,71 & $\mathrm{C}_{20} \mathrm{H}_{30}$ \\
\hline & & & & & & & $\mathrm{C}_{20} \mathrm{H}_{30}$ \\
\hline & 46,79 & 6,01 & 46,16 & 1,04 & 1,54 & 0,74 & \\
\hline $\begin{array}{l}\text { Semilla } \\
\text { chontaduro }\end{array}$ & 48,28 & 6.00 & 45,08 & 0.64 & 1.49 & 0.70 & $\mathrm{C}_{20} \mathrm{H}_{29,6} \mathrm{O}_{14,0} \mathrm{~N}_{0,16} \mathrm{~S}_{0,03}$ \\
\hline \multirow{2}{*}{$\begin{array}{l}\text { Cáscara } \\
\text { mamoncillo }\end{array}$} & & & & & & & $\mathrm{C}_{20} \mathrm{H}_{29,5} \mathrm{O}_{13,7} \mathrm{~N}_{0,20} \mathrm{~S}_{0,04}$ \\
\hline & 48,73 & 6,04 & 44,37 & 0,87 & 1,49 & 0,68 & \\
\hline $\begin{array}{l}\text { Semilla } \\
\text { mamoncillo }\end{array}$ & 48,5 & & 44, & & & & $\mathrm{C}_{20} \mathrm{H}_{29,4} \mathrm{O}_{13,9} \mathrm{~N}_{0,14} \mathrm{~S}_{0,03}$ \\
\hline Cáscara de sandía & 46,69 & 6,16 & 44,73 & 2,42 & $\begin{array}{l}1,40 \\
1,58\end{array}$ & 0,72 & $\mathrm{C}_{20} \mathrm{H}_{31,4} \mathrm{O}_{14,4} \mathrm{~N}_{0,75} \mathrm{~S}_{0}$ \\
\hline Semilla de sandía & 47,33 & 6,02 & 45,67 & 0,99 & 1,53 & 0,72 & \\
\hline Cáscara de papaya & 47,50 & 6,07 & 45,05 & 1,37 & 1,53 & 0,71 & \\
\hline Semillas de papaya & 46,83 & 6,07 & 45,54 & 1,56 & 1,56 & 0,73 & $\mathrm{C}_{20} \mathrm{H}_{30,8} \mathrm{O}_{14,6} \mathrm{~N}_{0,2}$ \\
\hline \multirow{2}{*}{$\begin{array}{ll}\text { Cáscara } & \text { de } \\
\text { durazno } & \end{array}$} & & & & & & & \\
\hline & 48,64 & 6,02 & 44,53 & 0,80 & 1,49 & 0,69 & \\
\hline Semilla de durazno & 48,66 & 6,00 & 44,82 & 0,53 & 1,48 & 0,69 & $\mathrm{C}_{20} \mathrm{H}_{29,3} \mathrm{O}_{13,8} \mathrm{~N}_{0,13} \mathrm{~S}_{0,02}$ \\
\hline \multirow{2}{*}{$\begin{array}{l}\text { Cáscara de arazá } \\
\text { Semilla de arazá }\end{array}$} & 49,44 & 6,00 & 44,15 & 0,40 & 1,46 & 0,67 & \\
\hline & 48,8 & 6,00 & 44,68 & 0,52 & 1,48 & 0,69 & \\
\hline $\begin{array}{l}\text { Semilla de arazá } \\
\text { Cáscara de curuba }\end{array}$ & 49,64 & 6,02 & 43,82 & 0,52 & 1,46 & 0,66 & \\
\hline Semilla de curuba & 49,94 & 5,99 & 43,90 & 0,17 & 1,44 & 0,66 & $\mathrm{C}_{20} \mathrm{H}_{28,5} \mathrm{O}_{13,2} \mathrm{~N}_{0,0}$ \\
\hline $\begin{array}{ll}\text { Cáscara } & \text { de } \\
\text { mangostino } & \end{array}$ & 50,65 & 5,99 & 43,32 & 0,04 & 1,42 & 0,64 & \\
\hline ang & & & & & & & $\mathrm{C}_{20} \mathrm{H}_{30,1} \mathrm{O}_{14,3} \mathrm{~N}_{0,23} \mathrm{~S}_{\mathrm{c}}$ \\
\hline mangostino & 47,64 & 6,03 & 45,30 & 1,03 & 1,52 & 0,71 & \\
\hline Semilla de guayaba & 48,39 & 6,00 & 45,00 & 0,61 & 1,49 & 0,70 & $\mathrm{C}_{20} \mathrm{H}$ \\
\hline Cáscara & & & & & & & \\
\hline granadilla & 47,3 & 6,04 & 45,43 & 1,18 & 1,53 & 0,72 & \\
\hline anadilla & & & & & & 069 & $\mathrm{C}_{20} \mathrm{H}_{29,3} \mathrm{O}_{13,7} \mathrm{~N}_{0,16} \mathrm{~S}_{0,02}$ \\
\hline Cáscara de banano & 47,52 & 6,10 & 44,67 & 1,71 & 1,54 & 0,71 & \\
\hline de & & & & & & & \\
\hline droño & 48,35 & 6,03 & 44,71 & 0,91 & 1,50 & 0,69 & \\
\hline de & & & & & & & $\mathrm{C}_{20} \mathrm{H}_{29,4} \mathrm{O}_{13,8} \mathrm{~N}_{0,14} \mathrm{~S}_{0}$ \\
\hline madroño & 48,5 & 6,00 & 44,93 & 0,57 & 1,48 & 0,69 & \\
\hline Cáscara de melón & 45,21 & 6,21 & 45,45 & 3,13 & 1,65 & 0,75 & \\
\hline Semilla de melón & 47,48 & 6,03 & 45,48 & 1,01 & 1,52 & 0,72 & \\
\hline de & & & & & & & $\mathrm{C}_{20} \mathrm{H}_{30,6} \mathrm{O}_{14,5} \mathrm{~N}_{0,42} \mathrm{~S}_{0}$ \\
\hline & 47,07 & 6,0 & & & 1,54 & 0,72 & \\
\hline Semilla de auyama & 47,04 & 6,03 & 45,77 & 1,16 & 1,54 & 0,73 & $\mathrm{C}_{20} \mathrm{H}_{30,5} \mathrm{O}_{14,6} \mathrm{~N}_{0,37} \mathrm{~S}_{0,02}$ \\
\hline
\end{tabular}

C: carbono, $\mathrm{H}$ : hidrógeno, O: oxígeno, N: nitrógeno, S: azufre, $\mathrm{H} / \mathrm{C}$ y O/C: relaciones atómicas.

Se agruparon los valores de $\mathrm{N}$ y $\mathrm{S}(\mathrm{N}+\mathrm{S})$ para analizar el potencial contaminante de los residuos respecto a las posibles emisiones generadas (NOx y SOx) por estos elementos al ser sometidos a combustión. Fuente: Propia de los autores.
A partir de las fórmulas empíricas de cada residuo y bajo condiciones de combustión completa, se encuentra que las mayores emisiones de $\mathrm{NO}_{3}$ y $\mathrm{SO}_{3}$, por cada mol de residuo, se presentan en el vástago de tomate de 
árbol $\left(0,70 \mathrm{~mol}\right.$ de $\mathrm{NO}_{3}$ y 0,05 moles de $\left.\mathrm{SO}_{3}\right)$, cáscara de sandía $\left(0,75 \mathrm{~mol}\right.$ de $\mathrm{NO}_{3}$ y 0,06 moles de $\left.\mathrm{SO}_{3}\right)$ y cáscara de melón $\left(1,10 \mathrm{~mol}\right.$ de $\mathrm{NO}_{3}$ y 0,04 moles de $\mathrm{SO}_{3}$ ). Mientras que las menores emisiones de $\mathrm{NO}_{3}$ y $\mathrm{SO}_{3}$, por cada mol de residuo, se presentan en la cáscara de mangostino $\left(0,01 \mathrm{~mol}\right.$ de $\mathrm{NO}_{3}$ y 0,01 moles de $\left.\mathrm{SO}_{3}\right)$, semilla de curuba $\left(0,04 \mathrm{~mol}\right.$ de $\mathrm{NO}_{3}$ y 0,01 moles de $\left.\mathrm{SO}_{3}\right)$, cáscara $\left(0,03 \mathrm{~mol}\right.$ de $\mathrm{NO}_{3}$ y 0,02 moles de $\left.\mathrm{SO}_{3}\right)$ y estopa de coco $\left(0,04 \mathrm{~mol}\right.$ de $\mathrm{NO}_{3}$ y 0,01 moles de $\left.\mathrm{SO}_{3}\right)$, y cáscara de naranja $(0,09$ mol de $\mathrm{NO}_{3}$ y 0,02 moles de $\mathrm{SO}_{3}$ ).

\section{Aprovechamientos de los residuos de frutas en combustión y pirólisis}

Para analizar el aprovechamiento potencial de los residuos de frutas como materia prima en combustión y pirólisis se realizaron los siguientes gráficos entre: poder calorífico superior (PCS) y contenido de cenizas (Cz) en la Fig. 1, PCS e índice de combustibilidad (MV/CF) en la Fig. 2, MV/CF y relación atómica $\mathrm{H} / \mathrm{C}$ en la Fig. 3, poder calorífico y relación atómica $\mathrm{O} / \mathrm{C}$ en la Fig. 4, y entre la relación atómica $\mathrm{H} / \mathrm{C}$ y la relación atómica O/C (diagrama de Van Krevelen) en la Fig. 5. En la Fig. 1 se observa que existe una relación lineal entre el $\mathrm{Cz}$ y el PCS. Este comportamiento es claro en las cáscaras de frutas, pero menos claro en las semillas. De igual manera, se encuentra que alrededor del $90 \%$ de las semillas tienen un PCS entre 17 y $19 \mathrm{MJ} / \mathrm{kg}$ con contenidos de $\mathrm{Cz}$ menores al 5\%, mientras que, para ese mismo porcentaje, las cáscaras presentan valores de PCS entre 17 y $20 \mathrm{MJ} / \mathrm{kg}$ con contenidos de $\mathrm{Cz}$ menores al $10 \%$. Esto indica que hay una mayor variación del contenido de $\mathrm{Cz}$ y PCS en las cáscaras que en las semillas de frutas.

También se aprecia que, en términos generales, las cáscaras de frutas tienen mayor PCS que las semillas. No obstante, se recomienda utilizar como combustible las semillas en lugar de las cáscaras, pues estas presentan menor porcentaje de $\mathrm{Cz}$, por lo cual se espera obtener menos incrustaciones en las paredes de la caldera, si las $\mathrm{Cz}$ son de carácter básico. Sin embargo, si las $\mathrm{Cz}$ son de carácter ácido, las $\mathrm{Cz}$ de las semillas producirán menor corrosión en las paredes de las calderas que las $\mathrm{Cz}$ producidas en la combustión de las cáscaras [8, 19]. Además, el aumento en el contenido de $\mathrm{Cz}$, afecta (disminuye) la velocidad de encendido en el proceso de combustión [20]. Asimismo, un menor contenido de cenizas hace que se tenga un menor volumen de material a disponer. Esto conlleva a reducir costos en disposición de residuos y en mantenimiento de las calderas [19]. De igual forma, para la pirólisis, se recomienda emplear residuos con menor contenido de cenizas, esto con el fin de que el biosólido obtenido en la pirólisis, no contenga cantidades considerables de $\mathrm{Cz}$ que provoquen problemas al utilizarlo como combustible [8, 19].

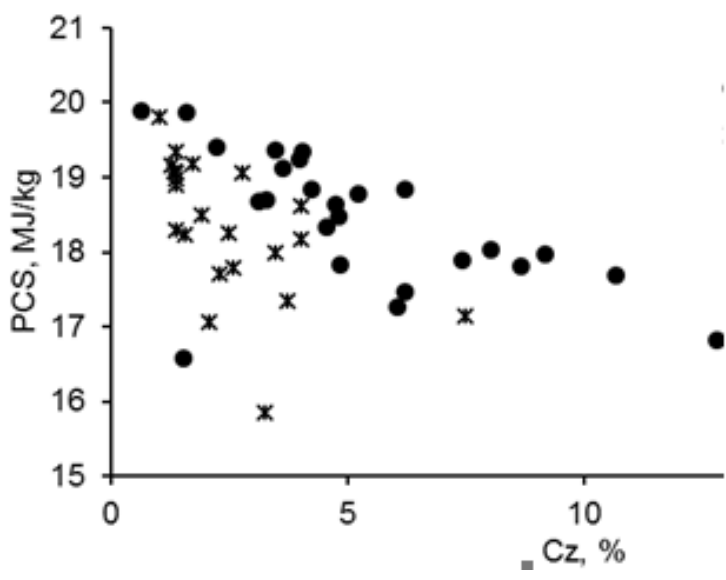

Fig. 1. Variación del poder calorífico superior con el contenido de ceniza de los residuos de frutas. Fuente: Propia de los autores.

En la Fig. 2 se presenta la variación del PCS con el IC de los residuos de frutas. Se observa que al aumentar el IC disminuye el PCS. Aun así, se esperaría que con el aumento en IC se mejore la reactividad en la combustión, generando una mayor estabilidad de la llama y reduciendo la temperatura de ignición del material combustible $[9,10]$. Según esto, las semillas de naranja, limón y auyama, y las cáscaras de chontaduro pueden ser considerados como materia prima para la generación de energía. En pirólisis, estos mismos residuos se recomiendan para la producción de una mayor fracción gaseosa, por su elevado contenido de materia volátil $[7,8]$. Si se desea obtener mayor contenido de biosólido, se recomienda emplear cáscara y semilla de curuba, y cáscaras de naranja, mandarina y mangostino. 


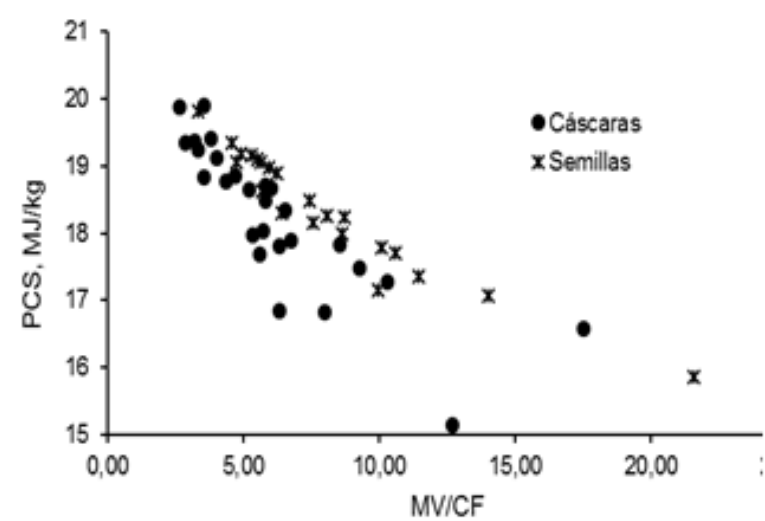

Fig. 2. Variación del poder calorífico superior con el índice de combustibilidad de los residuos de frutas. Fuente: propia de los autores

La variación del IC (MV/FC) con la relación atómica $\mathrm{H} / \mathrm{C}$ de los residuos de frutas se presenta en la Fig. 3. En la literatura se reporta que la materia volátil está formada por una parte combustible constituida por compuestos gaseosos $\mathrm{CxHy}$, $\mathrm{CO}$ e $\mathrm{H} 2$, por lo cual un mayor contenido de $\mathrm{H}$ en un residuo se ve reflejado en un alto contenido de MV [15]. Por lo tanto, para que un material sea reactivo a la combustión y favorezca la producción de la fracción gaseosa en el proceso de pirólisis, se requiere que el residuo tenga altos contenidos de $\mathrm{H}$ y $\mathrm{MV}$. Considerando esto, se confirma la conclusión de la Fig. 2, donde se recomiendan las semillas de naranja, limón y auyama, y las cáscaras de chontaduro. Además, un bajo IC y un bajo valor de la relación atómica $\mathrm{H} / \mathrm{C}$, indican que el residuo tiene mayor contenido de carbono fijo, lo cual permite tener una combustión más prolongada y mayor PCS, y en pirólisis se puede obtener una mayor fracción de biosólido [21].

En la Fig. 4 se muestra la variación del poder calorífico superior con la relación atómica $\mathrm{O} / \mathrm{C}$ de los residuos de frutas. Aquí se puede ver que una mayor relación atómica O/C hace que el PCS disminuya [21]. Esto indica que la presencia de oxígeno en el residuo no contribuye al PCS y dificulta la transformación de los residuos en combustibles líquidos en el proceso de pirólisis [10]. Esto se debe a que el oxígeno consume parte de hidrógeno para producir agua. Sin embargo, un alto contenido de hidrógeno y oxígeno en los residuos resulta en un mayor rendimiento de producción de volátiles y líquidos durante el proceso de pirólisis [7]. Igualmente, en esta figura se aprecia que, en general, las semillas tienen un mayor contenido de oxígeno que las cáscaras, confirmado los resultados mostrados en la Tabla 2. Este diagrama (PSC vs. $\mathrm{O} / \mathrm{C})$ también sirve para establecer comparaciones entre el PCS de diferentes residuos respecto al análisis último [21]. Allí se establece que los residuos con una alta relación atómica $\mathrm{O} / \mathrm{C}$ (mayor contenido de oxígeno y menor contenido de carbono) tienen un PCS menor que aquellos que tiene altos valores de las relaciones atómicas $\mathrm{O} / \mathrm{C}$ y H/C (mayor contenido de carbono) [21].

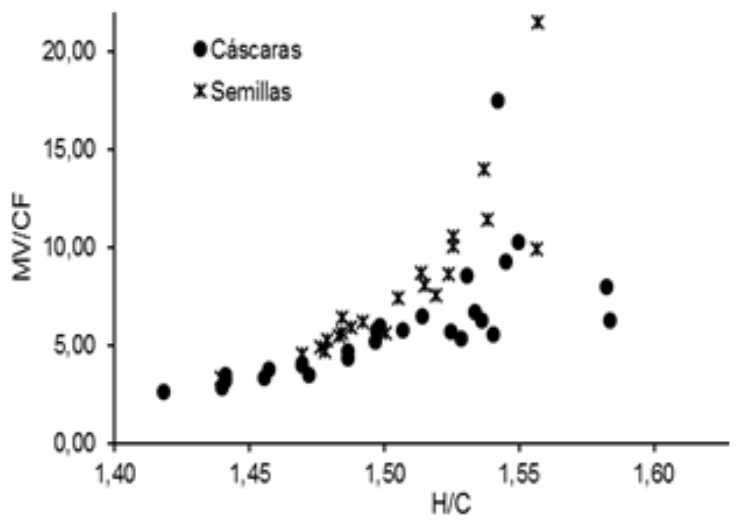

Fig. 3. Variación del índice de combustibilidad con la relación atómica $\mathrm{H} / \mathrm{C}$ de los residuos de frutas. Fuente: Propia de los autores.

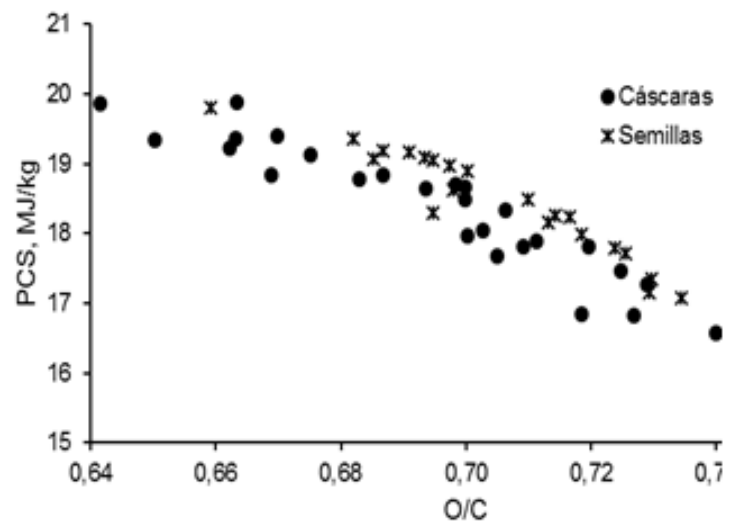

Fig. 4. Variación del poder calorífico superior con la relación atómica $\mathrm{O} / \mathrm{C}$ de los residuos de frutas. Fuente: Propia de los autores 
La correlación que existe entre las relaciones atómicas $\mathrm{H} / \mathrm{C}$ y $\mathrm{O} / \mathrm{C}$ para los residuos de frutas se analizan a través del diagrama de Van Krevelen que se presenta en la Fig. 5. El diagrama Van Krevelen se utiliza para establecer las diferencias en la composición elemental y PCS de diversos materiales de origen fósil o biomásico, o entre el mismo material que ha experimentado alguna transformación física o química [22]. En la figura se muestra encerrado en un círculo los residuos con menor PCS, que corresponden a la cáscara de melón $(15,15$ $\mathrm{MJ} / \mathrm{kg})$, semilla de naranja $(15,86 \mathrm{MJ} / \mathrm{kg})$, cáscara de chontaduro $(16,58 \mathrm{MJ} / \mathrm{kg})$ y semilla de limón $(17,07 \mathrm{MJ} / \mathrm{kg})$.

Como se puede ver en esta figura, tanto para las semillas $(R 2=0,96)$ como para las cáscaras $(R 2=0,89)$ la relación atómica $H / C$ se puede expresar como una función lineal de la relación atómica $\mathrm{O} / \mathrm{C}$ [23]. Analizando los coeficientes numéricos de las correlaciones presentadas en la Fig. 5, se encuentra hay diferencia entre las cáscaras y las semillas. Esto puede indicar que cada tipo de biomasa tiene su propia correlación característica. Esto se puede explicar debido a que las cáscaras y las semillas tienen diferentes contenidos orgánicos estructurales (celulosa, hemicelulosa y lignina), extractivos y minerales [24]. En general, las cáscaras presentan un alto contenido de celulosa y hemicelulosa, en cambio, las semillas tienen mayor porcentaje de lignina. En el caso de la pirólisis, los residuos con alto contenido de hemicelulosa producirán gases no condensables y baja cantidad de alquitrán, mientras que los residuos con mayor porcentaje de celulosa generarán gases condensables y una cantidad insignificante de carbón. Los residuos ricos en lignina producirán compuestos aromáticos y carbonizados, asimismo la pirólisis de la lignina contribuye al rendimiento del líquido, el cuál contendrá bioaceites y alquitrán [8].

La diferencia entre los contenidos estructurales de los residuos también explica los diferentes comportamientos entre los mismos tipos de estos. En la literatura Jones et al. [23] reporta la siguiente correlación para un gran rango de biomasas:

$\frac{H}{C}=1,4125 \frac{O}{C}+0,5004$

(5)
Al comparar estas ecuaciones se observa que la correlación de las semillas se ajusta más a lo reportado por Jones et al. [23].

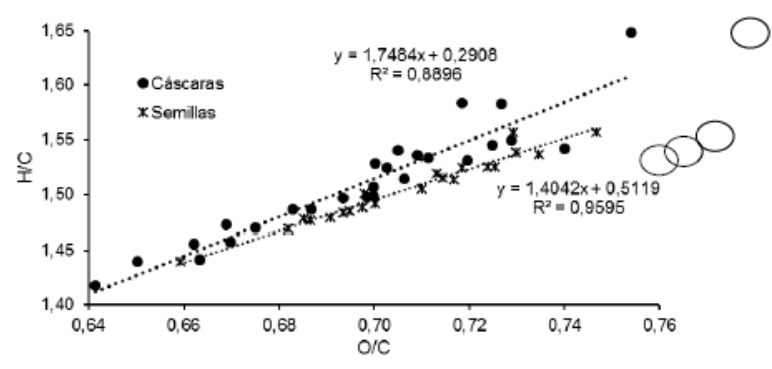

Fig. 5. Diagrama de Van Krevelen para los residuos de frutas. Donde $y=H / C$ y $x=O / C$. Fuente: Propia de los autores.

\section{CONCLUSIONES}

Los residuos de frutas representan una fuente potencial de energía. Si se considera el poder calorífico superior de los residuos de fruta analizados, se esperaría que su uso como materia prima para la obtención de energía no solo mitigue el impacto ambiental negativo debido a su disposición inadecuada, sino que también genere una mejora en la economía. Según el contenido de cenizas de los residuos de fruta analizados, se propone utilizar semillas de frutas en procesos de combustión, ya que estas generarán menor corrosión o incrustaciones. Asimismo, estos residuos se pueden aplicar en pirólisis con el fin de obtener un biosólido con mejores propiedades. Las semillas de naranja, limón y auyama, y las cáscaras de chontaduros se caracterizan por presentar un alto IC, por lo que se recomiendan para procesos de combustión con mayor estabilidad de la llama y menor temperatura de ignición del material combustible. Estos mismos residuos presentan un alto contenido de materia volátil e hidrógeno, por lo que tienen aplicaciones en procesos de pirólisis en los que se desee obtener una mayor fracción gaseosa o líquida. Por otro lado, si se busca una combustión prolongada o una mayor fracción de biosólido en pirólisis, se sugiere utilizar residuos como las cáscaras de mangostino, naranja, curuba, guanábana, coco y arazá, estopa de coco y semilla de curuba, los cuales presentan un bajo IC y baja relación atómica $\mathrm{H} / \mathrm{C}$. 


\section{AGRADECIMIENTOS}

Los autores desean agradecer a la Universidad Nacional de Colombia por el apoyo al proyecto "Identificación, cuantificación y caracterización de los residuos orgánicos generados en la agroindustria caldense para su aprovechamiento energético y bioactivo" con código HERMES 35961.

\section{REFERENCIAS BIBLIOGRÁFICAS}

[1] Peñaranda, L. V., Montenegro, S. P. y Giraldo, P. A. Aprovechamiento de residuos agroindustriales en Colombia. Revista de Investigación Agraria y Ambiental, 8(2), 141-150, 2017.

[2] Díaz, A. L. Biodegradación de residuos de frutas $y$ vegetales provenientes de supermercados usando la técnica de aireación forzada, Kuxulkab - Revista de divulgación, 17 (33), 5-7, 2011.

[3] Vargas, Y. A. y Pérez, L. I. Aprovechamiento de residuos agroindustriales en el mejoramiento de la calidad del ambiente, Revista Facultad de Ciencias Básicas, 14 (1), 1-14, 2018.

[4] Iglesias, D. Costos económicos por la generación y manejo de residuos sólidos en el municipio de Toluca, Estado de México. Revista Equilibrio Económico, 3 (2), 131-148, 2007.

[5] Oro, A., Da Silva, E., Da Silva, D. A., Hillig, E. y Pelz, S. K. Biomass energetic potential from timber harvesting at different times of storage, Floresta, 48 (1), 9-18, 2018.

[6] Pantoja-Chamorro, A. L., Hurtado-Benavides, A. M. y Martínez-Correa, H. A. Caracterización de aceite de semillas de maracuyá (Passiflora edulis Sims.) procedentes de residuos agroindustriales obtenido con $\mathrm{CO} 2$ supercrítico, Acta Agronómica, 66 (2), 178-185, 2017.

[7] Demirbas, A. Fuels from biomass. Biorefineries for biomass upgrading facilities, Springer, Londres, 2010.

[8] Basu, P. Biomass gasification, pyrolysis and torrefaction: practical design and theory. Elsevier, Burlington, 2013.

[9] Prieto, A. y Chaves, A. Aplicación de métodos termogravimétricos y su correlación $\mathrm{C} / \mathrm{O}$ y $\mathrm{C} / \mathrm{H}$ en la combustión de carbón en calderas de parrilla móvil. Santiago de Cali, Universidad del Valle, 1998.

[10] Urhan, R. M. Mejora de la eficiencia de la combustión de carbones utilizados en el Valle del Cauca mediante un estudio previo de su reactividad, Santiago de Cali, Universidad del Valle - UPB, 2000.

[11] Rivas-Ubach, A., Lui, Y., Bianchi, T. S., Tolic, N., Jansson, C. y Pasa-Tolic, L. Moving beyond the van Krevelen Diagram: A New Stoichiometric Approach for Compound Classification in Organisms, Anal. Chem, 90 (10), 6152-6160, 2018.

[12] Cai, J., He, Y., Yu, X., Banks, S. W., Yang, Y., Zhang, X., Yu, Y., Liu, R. y Bridgwater, A. V. Review of physicochemical properties and analytical characterization of lignocellulosic biomass, Renewable and Sustainable Energy Reviews, 76, 309-322, 2017.

[13] Wang, C., Zhu, X., Liu, X., Lv, Q., Zhao, L. y Che, D. Correlations of chemical properties of high-alkali solid fuels: A comparative study between Zhundong coal and biomass, Fuel, 211, 629-637, 2018.

[14] Cámara, A. Diseño y puesta a punto de un protocolo para determinar el poder calorífico de sólidos. Aplicación a residuos plásticos. Trabajo fin de grado en Ingeniería en Tecnologías Industriales. Universitat Politécnica de Valéncia. 2017.

[15] García, R., Pizarro, C., Lavín, A.G. y Bueno, J.L. Spanish biofuels heating value estimation. Part II: Proximate analysis data, Fuel, 117, 1139_ 1147, 2014.

[16] Rincón, J.M. y Silva, E.E. Bioenergía: Fuentes, conversión y sustentabilidad, Charlie's Impresores Ltda., Bogotá, 2015.

[17] Vargas, Y.A. y Pérez, L.I. Aprovechamiento de residuos agroindustriales para el mejoramiento de la calidad del ambiente, Revista Facultad de Ciencias Básicas, 14 (1), 59-72, 2018.

[18] Cadavid, L.S. y Bolaños, I.V. Aprovechamiento de residuos orgánicos para la producción de energía renovable en una ciudad colombiana, Energética, 46, 23-28, 2015. 
[19] Nunes, L.J.R., Matias, J.C.O. y Catalão J.P.S. Biomass combustion systems: A review on the physical and chemical properties of the ashes, Renewable and Sustainable Energy Reviews, 53, 235-242, 2016.

[20] Boumanchar, I., Chhiti, Y., M'hamdi, F. E., El, A., Sahibed-Dine, A., Bentiss, F., Jama, C. y Bensitel, M., Effect of materials mixture on the higher heating value: Case of biomass, biochar and municipal solid Waste, Waste Management, 61, 78-86, 2017.

[21] Arteaga-Perez, L.E., Flores, M., Escobar, M., Segura, C. y Gordon, A. Análisis comparativo de la torrefacción húmeda y seca de Pinus radiata, Energética, 46, 5-12, diciembre 2015.

[22] Van der Stelt, M.J.C. Gerhauser, H., Kiel, M.J.C. y Ptasinski, K.J. Biomass upgrading by torrefaction for the production of biofuels: A review, Biomass and Bioenergy, 35, 3748-3762, 2011.

[23] Jones, J. M., Nawaz, M., Darvell, L. I., Ross, A. B., Pourkashanian, M. y Williams, A. Towards biomass classification for energy applications, Science in Thermal and Chemical Biomass Conversion, Victoria, Canadá, 331-339, agostoseptiembre 2004.

[24] Vassilev, S.V., Baxter, D., Andersen, L.K., Vassileva, C.G. y Morgan, T.J. An overview of the organic and inorganic phase composition of biomass, Fuel, 94, 1-33, 2012. 